\title{
Modified Landau levels, damped harmonic oscillator, and two-dimensional pseudo-bosons
}

S. Twareque Ali, F. Bagarello, and Jean Pierre Gazeau

Citation: J. Math. Phys. 51, 123502 (2010); doi: 10.1063/1.3514196

View online: http://dx.doi.org/10.1063/1.3514196

View Table of Contents: http://jmp.aip.org/resource/1/JMAPAQ/v51/i12

Published by the American Institute of Physics.

\section{Related Articles}

Uncertainty relation for non-Hamiltonian quantum systems

J. Math. Phys. 54, 012112 (2013)

Fractional Schrödinger equation for a particle moving in a potential well

J. Math. Phys. 54, 012111 (2013)

Off-shell Jost solutions for Coulomb and Coulomb-like interactions in all partial waves

J. Math. Phys. 54, 013514 (2013)

Generalized Kepler problems. I. Without magnetic charges

J. Math. Phys. 54, 012109 (2013)

High-energy analysis and Levinson's theorem for the selfadjoint matrix Schrödinger operator on the half line J. Math. Phys. 54, 012108 (2013)

\section{Additional information on J. Math. Phys.}

Journal Homepage: http://jmp.aip.org/

Journal Information: http://jmp.aip.org/about/about_the_journal

Top downloads: http://jmp.aip.org/features/most_downloaded

Information for Authors: http://jmp.aip.org/authors

\section{ADVERTISEMENT}

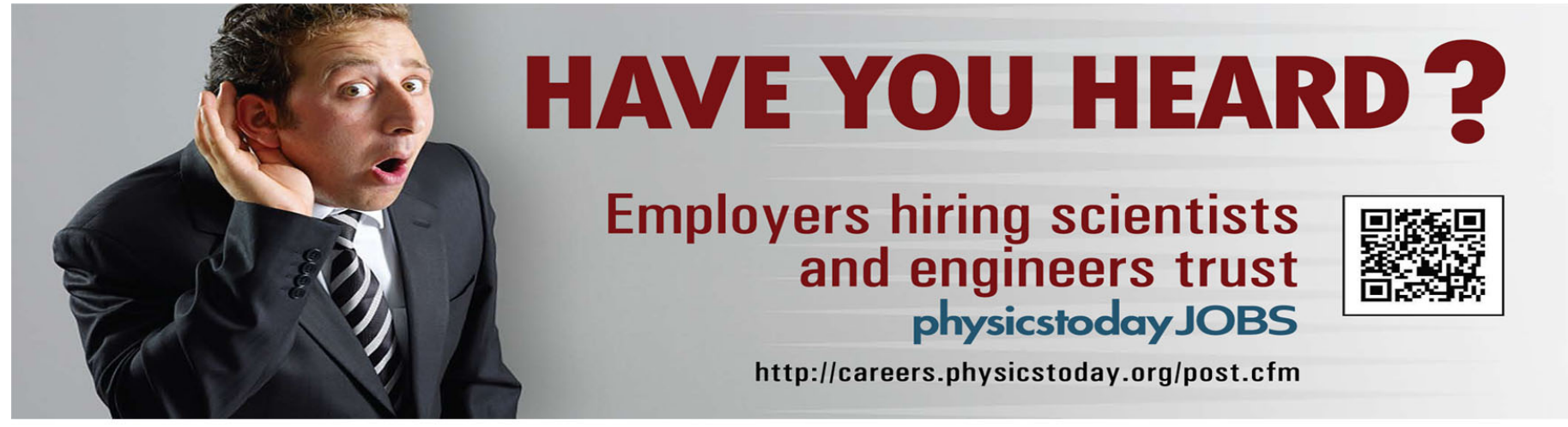




\title{
Modified Landau levels, damped harmonic oscillator, and two-dimensional pseudo-bosons
}

\author{
S. Twareque Ali, ${ }^{1}$ F. Bagarello, ${ }^{2, a)}$ and Jean Pierre Gazeau ${ }^{3}$ \\ ${ }^{1}$ Department of Mathematics and Statistics, Concordia University, Montréal, Québec, \\ Canada H3G 1 M8 \\ ${ }^{2}$ Dipartimento di Metodi e Modelli Matematici, Facoltà di Ingegneria, Università di Palermo, \\ I-90128 Palermo, Italy \\ ${ }^{3}$ Laboratoire APC, Université Paris 7-Denis Diderot, 10, rue A. Domon et L. Duquet, 75205 \\ Paris Cedex 13, France
}

(Received 27 July 2010; accepted 19 October 2010; published online 2 December 2010)

In a series of recent papers, one of us has analyzed in some details a class of elementary excitations called pseudo-bosons. They arise from a special deformation of the canonical commutation relation $\left[a, a^{\dagger}\right]=\mathbb{1}$, which is replaced by $[a, b]=\mathbb{1}$, with $b$ not necessarily equal to $a^{\dagger}$. Here, after a two-dimensional extension of the general framework, we apply the theory to a generalized version of the two-dimensional Hamiltonian describing Landau levels. Moreover, for this system, we discuss coherent states and we deduce a resolution of the identity. We also consider a different class of examples arising from a classical system, i.e., a damped harmonic oscillator. @ 2010 American Institute of Physics. [doi:10.1063/1.3514196]

\section{INTRODUCTION}

In a series of recent papers ${ }^{1-4}$ one of us (FB) has investigated some mathematical aspects of the so-called pseudo-bosons, originally introduced by Trifonov in Ref. 5. They arise from the canonical commutation relation $\left[a, a^{\dagger}\right]=\mathbb{1}$ upon replacing $a^{\dagger}$ by another (unbounded) operator $b$ not (in general) related to $a:[a, b]=11$. We have shown that $N=b a$ and $N^{\dagger}=a^{\dagger} b^{\dagger}$ can be both diagonalized and that their spectra coincide with the set of natural numbers (including 0 ), $\mathbb{N}_{0}$. However, the sets of related eigenvectors are not orthonormal bases, but, nevertheless, they are automatically biorthogonal. In all the examples considered so far, they are bases of the Hilbert space of the system, $\mathcal{H}$, and, in some cases, they turn out to be Riesz bases.

To our knowledge, not many physical consequences of this construction have been discussed up to now. For this reason, extending what two of us (STA and FB) have already done in Ref. 6, we will construct here a two-dimensional (2D) model which fits the main assumptions of the construction given in Ref. 1 and which is physically motivated. We will further consider a second example, again physically motivated, arising from the quantization of the damped harmonic oscillator. ${ }^{7}$

This paper is organized as follows. In Sec. II, we introduce and discuss 2D pseudo-bosons analyzing some of their mathematical properties and their related coherent states. In Sec. III, we introduce the generalized Landau levels (GLL) and we discuss them in the context of Sec. II. $\mathrm{Sec}$. IV is devoted to our analysis of the quantum damped harmonic oscillator, while Sec. V contains our conclusions.

\section{THE COMMUTATION RULES}

In this section, we will construct a $2 \mathrm{D}$ version of what originally proposed in Ref. 1, to which we refer for further comments on the 1D situation.

\footnotetext{
a)Electronic mail: bagarell@unipa.it.
} 
Let $\mathcal{H}$ be a given Hilbert space with scalar product $\langle.,$.$\rangle and related norm \|$.$\| . We introduce two$ pairs of operators, $a_{j}$ and $b_{j}, j=1,2$, acting on $\mathcal{H}$ and satisfying the following commutation rules:

$$
\left[a_{j}, b_{j}\right]=11, \quad \text { and } \quad\left[a_{1}^{\sharp}, a_{2}^{\sharp}\right]=\left[a_{1}^{\sharp}, b_{2}^{\sharp}\right]=\left[b_{1}^{\sharp}, b_{2}^{\sharp}\right]=0,
$$

where $x^{\sharp}$ stands for $x$ or $x^{\dagger}\left(x=a_{j}, b_{j}\right)$. Of course, they collapse to the canonical commutation relations for independent modes if $b_{j}=a_{j}^{\dagger}, j=1,2$. It is well known that $a_{j}$ and $b_{j}$ are unbounded operators, so they cannot be defined on all of $\mathcal{H}$. Following Ref. 1, and writing $D^{\infty}(X):=\cap_{p \geq 0} D\left(X^{p}\right)$ (the common domain of all the powers of the operator $X$ ), we consider the following:

Assumption 1. There exists a non-zero $\varphi_{0,0} \in \mathcal{H}$ such that $a_{j} \varphi_{0,0}=0, j=1,2$, and $\varphi_{0,0} \in D^{\infty}\left(b_{1}\right) \cap D^{\infty}\left(b_{2}\right)$.

Assumption 2. There exists a non-zero $\Psi_{0,0} \in \mathcal{H}$ such that $b_{j}^{\dagger} \Psi_{0,0}=0, j=1,2$, and $\Psi_{0,0} \in D^{\infty}\left(a_{1}^{\dagger}\right) \cap D^{\infty}\left(a_{2}^{\dagger}\right)$.

Under these assumptions, we can introduce the following vectors in $\mathcal{H}$ :

$$
\varphi_{n, l}=\frac{1}{\sqrt{n ! l !}} b_{1}^{n} b_{2}^{l} \varphi_{0,0} \quad \text { and } \quad \Psi_{n, l}=\frac{1}{\sqrt{n ! l !}}\left(a_{1}^{\dagger}\right)^{n}\left(a_{2}^{\dagger}\right)^{l} \Psi_{0,0}, \quad n, l \geq 0 .
$$

Let us now define the unbounded operators $N_{j}:=b_{j} a_{j}$ and $\mathfrak{N}_{j}:=N_{j}^{\dagger}=a_{j}^{\dagger} b_{j}^{\dagger}, j=1,2$. It is possible to check that $\varphi_{n, l}$ belongs to the domain of $N_{j}, D\left(N_{j}\right)$, and $\Psi_{n, l} \in D\left(\mathfrak{N}_{j}\right)$, for all $n, l \geq 0$ and $j=1,2$. Moreover,

$$
N_{1} \varphi_{n, l}=n \varphi_{n, l}, \quad N_{2} \varphi_{n, l}=l \varphi_{n, l}, \quad \mathfrak{N}_{1} \Psi_{n, l}=n \Psi_{n, l}, \quad \mathfrak{N}_{2} \Psi_{n, l}=l \Psi_{n, l} .
$$

Under the above assumptions, it is easy to check that $\left\langle\Psi_{n, l}, \varphi_{m, k}\right\rangle=\delta_{n, m} \delta_{l, k}\left\langle\Psi_{0,0}, \varphi_{0,0}\right\rangle$ for all $n, m, l, k \geq 0$, which, if we choose the normalization of $\Psi_{0,0}$ and $\varphi_{0,0}$ in such a way that $\left\langle\Psi_{0,0}, \varphi_{0,0}\right\rangle=1$, becomes

$$
\left\langle\Psi_{n, l}, \varphi_{m, k}\right\rangle=\delta_{n, m} \delta_{l, k}, \quad \forall n, m, l, k \geq 0 .
$$

This means that the sets $\mathcal{F}_{\Psi}=\left\{\Psi_{n, l}, n, l \geq 0\right\}$ and $\mathcal{F}_{\varphi}=\left\{\varphi_{n, l}, n, l \geq 0\right\}$ are biorthogonal and, because of this, the vectors of each set are linearly independent. If we now call $\mathcal{D}_{\varphi}$ and $\mathcal{D}_{\Psi}$, respectively, the linear span of $\mathcal{F}_{\varphi}$ and $\mathcal{F}_{\Psi}$, and $\mathcal{H}_{\varphi}$ and $\mathcal{H}_{\Psi}$ their closures, then

$$
f=\sum_{n, l=0}^{\infty}\left\langle\Psi_{n, l}, f\right\rangle \varphi_{n, l}, \quad \forall f \in \mathcal{H}_{\varphi}, \quad h=\sum_{n, l=0}^{\infty}\left\langle\varphi_{n, l}, h\right\rangle \Psi_{n, l}, \quad \forall h \in \mathcal{H}_{\Psi} .
$$

What is not in general ensured is that the Hilbert spaces introduced so far all coincide, i.e., that $\mathcal{H}_{\varphi}=\mathcal{H}_{\Psi}=\mathcal{H}$. Indeed, we can only state that $\mathcal{H}_{\varphi} \subseteq \mathcal{H}$ and $\mathcal{H}_{\Psi} \subseteq \mathcal{H}$. However, motivated by the examples already discussed in the literature and anticipating the discussion in Sec. III, we make the following assumption.

Assumption 3. The above Hilbert spaces all coincide: $\mathcal{H}_{\varphi}=\mathcal{H}_{\Psi}=\mathcal{H}$, which was introduced in Ref. 1 . This means, in particular, that both $\mathcal{F}_{\varphi}$ and $\mathcal{F}_{\Psi}$ are bases of $\mathcal{H}$. Let us now introduce the operators $S_{\varphi}$ and $S_{\Psi}$ via their action, respectively, on $\mathcal{F}_{\Psi}$ and $\mathcal{F}_{\varphi}$ :

$$
S_{\varphi} \Psi_{n, k}=\varphi_{n, k}, \quad S_{\Psi} \varphi_{n, k}=\Psi_{n, k},
$$

for all $n, k \geq 0$, which also imply that $\Psi_{n, k}=\left(S_{\Psi} S_{\varphi}\right) \Psi_{n, k}$ and $\varphi_{n, k}=\left(S_{\varphi} S_{\Psi}\right) \varphi_{n, k}$, for all $n, k \geq 0$. Hence,

$$
S_{\Psi} S_{\varphi}=S_{\varphi} S_{\Psi}=11 \quad \Rightarrow \quad S_{\Psi}=S_{\varphi}^{-1}
$$

In other words, both $S_{\Psi}$ and $S_{\varphi}$ are invertible and one is the inverse of the other. Furthermore, we can also check that they are both positive, well defined, and symmetric. ${ }^{1}$ Moreover, at least formally, it is possible to write these operators in the bra-ket notation as

$$
S_{\varphi}=\sum_{n, k=0}^{\infty}\left|\varphi_{n, k}><\varphi_{n, k}\right|, \quad S_{\Psi}=\sum_{n, k=0}^{\infty}\left|\Psi_{n, k}><\Psi_{n, k}\right| .
$$


These expressions are only formal, at this stage, since the series may not converge in the uniform topology and the operators $S_{\varphi}$ and $S_{\Psi}$ could be unbounded. This aspect was exhaustively discussed in Ref. 1, where the role of Riesz bases, ${ }^{19}$ in relation with the boundedness of $S_{\varphi}$ and $S_{\Psi}$, has been discussed in detail. We shall come back to this aspect later. However, we shall not assume here, except when explicitly stated, what has been called Assumption 4 in Ref. 1, since in most examples considered so far, and in what we are going to discuss in Sec. III, this assumption is not satisfied.

It is interesting to remark that, as in Ref. 1, even these 2D pseudo-bosons give rise to interesting intertwining relations among non-self-adjoint operators (see Ref. 3 and references therein). In particular, it is easy to check that

$$
S_{\Psi} N_{j}=\mathfrak{N}_{j} S_{\Psi} \quad \text { and } \quad N_{j} S_{\varphi}=S_{\varphi} \mathfrak{N}_{j},
$$

$j=1,2$. This is related to the fact that the spectra of, say, $N_{1}$ and $\mathfrak{N}_{1}$ coincide and that their eigenvectors are related by the operators $S_{\varphi}$ and $S_{\Psi}$, in agreement with the literature on intertwining operators $^{8,9}$ and on pseudo-Hermitian quantum mechanics (see Ref. 10 and references therein).

\section{A. Coherent states}

As it is well known, there exist several different, and not always equivalent, ways to define coherent states. ${ }^{11,12}$ In this paper, we will adopt the following definition, generalizing Ref. 1 . Let $z_{1}$ and $z_{2}$ be two complex variables, $z_{1}, z_{2} \in \mathcal{D}$ (some domain in $\mathbb{C}$ ), and let us introduce the following operators:

$$
U_{j}\left(z_{j}\right)=e^{z_{j} b_{j}-\bar{z}_{j} a_{j}}=e^{-\left|z_{j}\right|^{2} / 2} e^{z_{j} b_{j}} e^{-\bar{z}_{j} a_{j}}, \quad V_{j}\left(z_{j}\right)=e^{z_{j} a_{j}^{\dagger}-\bar{z}_{j} b_{j}^{\dagger}}=e^{-\left|z_{j}\right|^{2} / 2} e^{z_{j} a_{j}^{\dagger}} e^{-\bar{z}_{j} b_{j}^{\dagger}},
$$

$j=1,2$, and

$$
U\left(z_{1}, z_{2}\right):=U_{1}\left(z_{1}\right) U_{2}\left(z_{2}\right), \quad V\left(z_{1}, z_{2}\right):=V_{1}\left(z_{1}\right) V_{2}\left(z_{2}\right)
$$

and the following vectors:

$$
\varphi\left(z_{1}, z_{2}\right)=U\left(z_{1}, z_{2}\right) \varphi_{0,0}, \quad \Psi\left(z_{1}, z_{2}\right)=V\left(z_{1}, z_{2}\right) \Psi_{0,0}
$$

Remarks. (1) Due to the commutation rules for the operators $b_{j}$ and $a_{j}$, we clearly have $\left[U_{1}\left(z_{1}\right), U_{2}\left(z_{2}\right)\right]=\left[V_{1}\left(z_{1}\right), V_{2}\left(z_{2}\right)\right]=0$.

(2) Since the operators $U$ and $V$ are, for generic $z_{1}$ and $z_{2}$, unbounded, definition (2.12) makes sense only if $\varphi_{0,0} \in D(U)$ and $\Psi_{0,0} \in D(V)$, a condition which will be assumed here. In Ref. 1, it was proved that, for instance, this is so when $\mathcal{F}_{\varphi}$ and $\mathcal{F}_{\Psi}$ are Riesz bases.

(3) The set $\mathcal{D}$ could, in principle, be a proper subset of $\mathbb{C}$.

It is possible to write the vectors $\varphi\left(z_{1}, z_{2}\right)$ and $\Psi\left(z_{1}, z_{2}\right)$ in terms of the vectors of $\mathcal{F}_{\Psi}$ and $\mathcal{F}_{\varphi}$ as

$$
\varphi\left(z_{1}, z_{2}\right)=e^{-\left(\left|z_{1}\right|^{2}+\left|z_{2}\right|^{2}\right) / 2} \sum_{n, l=0}^{\infty} \frac{z_{1}^{n} z_{2}^{l}}{\sqrt{n ! l !}} \varphi_{n, l}, \quad \Psi\left(z_{1}, z_{2}\right)=e^{-\left(\left|z_{1}\right|^{2}+\left|z_{2}\right|^{2}\right) / 2} \sum_{n, l=0}^{\infty} \frac{z_{1}^{n} z_{2}^{l}}{\sqrt{n ! l !}} \Psi_{n, k} .
$$

These vectors are called coherent since they are eigenstates of the lowering operators. Indeed we can check that

$$
a_{j} \varphi\left(z_{1}, z_{2}\right)=z_{j} \varphi\left(z_{1}, z_{2}\right), \quad b_{j}^{\dagger} \Psi\left(z_{1}, z_{2}\right)=z_{j} \Psi\left(z_{1}, z_{2}\right),
$$

for $j=1,2$ and $z_{j} \in \mathcal{D}$. It is also a standard exercise, putting $z_{j}=r_{j} e^{i \theta_{j}}$, to check that the following operator equalities hold:

$$
\begin{aligned}
& \frac{1}{\pi^{2}} \int_{\mathbb{C}} d z_{1} \int_{\mathbb{C}} d z_{2}\left|\varphi\left(z_{1}, z_{2}\right)><\varphi\left(z_{1}, z_{2}\right)\right|=S_{\varphi}, \\
& \frac{1}{\pi^{2}} \int_{\mathbb{C}} d z_{1} \int_{\mathbb{C}} d z_{2}\left|\Psi\left(z_{1}, z_{2}\right)><\Psi\left(z_{1}, z_{2}\right)\right|=S_{\Psi},
\end{aligned}
$$


as well as

$$
\frac{1}{\pi^{2}} \int_{\mathbb{C}} d z_{1} \int_{\mathbb{C}} d z_{2}\left|\varphi\left(z_{1}, z_{2}\right)><\Psi\left(z_{1}, z_{2}\right)\right|=\frac{1}{\pi^{2}} \int_{\mathbb{C}} d z_{1} \int_{\mathbb{C}} d z_{2}\left|\Psi\left(z_{1}, z_{2}\right)><\varphi\left(z_{1}, z_{2}\right)\right|=\mathbb{1},
$$

which are written in convenient bra-ket notation. It should be said that these equalities are, most of the times, only formal results. Indeed it is not difficult to construct examples in which something goes wrong and, for instance, the resolution of the identity for the pair $\varphi\left(z_{1}, z_{2}\right)$ and $\Psi\left(z_{1}, z_{2}\right)$ does not hold as expected. As the following theorem implies, this is a reflection of the fact that the operators $S_{\varphi}$ and $S_{\Psi}$ are unbounded, or, equivalently, of the fact that $\mathcal{F}_{\varphi}$ and $\mathcal{F}_{\Psi}$ are not Riesz bases. Indeed we have the following general result, which was essentially stated in Ref. 2 for a concrete example of 1D pseudo-bosons, and which we extend here to the general setting.

Theorem 1. Let $a_{j}, b_{j}, \mathcal{F}_{\varphi}, \mathcal{F}_{\Psi}, \varphi\left(z_{1}, z_{2}\right)$ and $\Psi\left(z_{1}, z_{2}\right)$ be as above. Let us assume that (1) $\mathcal{F}_{\varphi}, \mathcal{F}_{\Psi}$ are Riesz bases; (2) $\mathcal{F}_{\varphi}, \mathcal{F}_{\Psi}$ are biorthogonal. Then (2.16) holds true.

The proof of this theorem does not differ significantly from that given in Ref. 2, so that it will not be repeated here. The meaning of the theorem is the following: suppose that following the above construction the coherent states we get do not produce a resolution of the identity. Then, since $\mathcal{F}_{\varphi}$ and $\mathcal{F}_{\Psi}$ are automatically biorthogonal, they cannot be Riesz bases (neither one of them)! However, this theorem does not hold in general for other types of coherent states. We will come back on this point in the next section.

\section{GENERALIZED LANDAU LEVELS}

The Hamiltonian of a single electron, moving on a 2D plane and subject to a uniform magnetic field along the $z$-direction, is given by the operator

$$
H_{0}^{\prime}=\frac{1}{2}(\underline{p}+\underline{A}(r))^{2}=\frac{1}{2}\left(p_{x}-\frac{y}{2}\right)^{2}+\frac{1}{2}\left(p_{y}+\frac{x}{2}\right)^{2},
$$

where we have used minimal coupling and the symmetric gauge $\vec{A}=\frac{1}{2}(-y, x, 0)$.

The spectrum of this Hamiltonian is easily obtained by first introducing the new variables

$$
P_{0}^{\prime}=p_{x}-y / 2, \quad Q_{0}^{\prime}=p_{y}+x / 2 .
$$

In terms of $P_{0}^{\prime}$ and $Q_{0}^{\prime}$, the single electron Hamiltonian, $H_{0}^{\prime}$, can be rewritten as

$$
H_{0}^{\prime}=\frac{1}{2}\left(Q_{0}^{\prime 2}+P_{0}^{\prime 2}\right)
$$

On a classical level, the transformation (3.2) is part of a canonical map from the phase space variables $\left(x, y, p_{x}, p_{y}\right)$ to $\left(Q_{0}, P_{0}, Q_{0}^{\prime}, P_{0}^{\prime}\right)$, where

$$
P_{0}=p_{y}-x / 2, \quad Q_{0}=p_{x}+y / 2,
$$

which can be used to construct a second Hamiltonian $H_{0}=\frac{1}{2}\left(Q_{0}^{2}+P_{0}^{2}\right)$.

The corresponding quantized operators satisfy the commutation relations:

$$
\left[x, p_{x}\right]=\left[y, p_{y}\right]=i, \quad\left[x, p_{y}\right]=\left[y, p_{x}\right]=[x, y]=\left[p_{x}, p_{y}\right]=0,
$$

and

$$
\left[Q_{0}, P_{0}\right]=\left[Q_{0}^{\prime}, P_{0}^{\prime}\right]=i, \quad\left[Q_{0}, P_{0}^{\prime}\right]=\left[Q_{0}^{\prime}, P_{0}\right]=\left[Q_{0}, Q_{0}^{\prime}\right]=\left[P_{0}, P_{0}^{\prime}\right]=0,
$$

so that $\left[H_{0}, H_{0}^{\prime}\right]=0$.

We refer to Ref. 13 and references therein for a discussion on how the corresponding wave functions look in different representations. In Ref. 6, two of us (STA and FB) have considered, in the context of supersymmetric (SUSY) quantum mechanics, an extended version of $H_{0}^{\prime}$, an extension needed due to the fact that for the Hamiltonian of the standard Landau levels (SLLs), there is essentially no difference between $H_{0}^{\prime}$ and its SUSY partner Hamiltonian. 
The extension constructed in Ref. 6 is very natural and simple: introducing the vector valued function $\vec{W}_{0}=-\frac{1}{2}(x, y, 0)=\left(W_{0,1}, W_{0,2}, 0\right)$, we may rewrite the operators in (3.2) and (3.4) as

$$
P_{0}^{\prime}=p_{x}+W_{0,2}, \quad Q_{0}^{\prime}=p_{y}-W_{0,1}, \quad P_{0}=p_{y}+W_{0,1}, \quad Q_{0}=p_{x}-W_{0,2} .
$$

This definition was extended in Ref. 6 as follows:

$$
p^{\prime}=p_{x}+W_{2}, \quad q^{\prime}=p_{y}-W_{1}, \quad p=p_{y}+W_{1}, \quad q=p_{x}-W_{2},
$$

introducing a vector superpotential $\vec{W}=\left(W_{1}, W_{2}, 0\right)$.

Here, since we are interested in constructing 2D pseudo-bosons, it is convenient to introduce two (in general) complex and different vector superpotentials (this is a slight abuse of language!) $\vec{W}=\left(W_{1}, W_{2}\right)$ and $\vec{V}=\left(V_{1}, V_{2}\right)$, and we put

$$
P^{\prime}=p_{x}+W_{2}, \quad Q^{\prime}=p_{y}-W_{1}, \quad P=p_{y}+V_{1}, \quad Q=p_{x}-V_{2} .
$$

Our notation is the following: all operators with suffix 0 are related to the SLL. The same operators, without the 0 , have to do with our generalized model, i.e., with the GLL. Notice that these operators are, in general, not self-adjoint. Hence, while for example $P_{0}=P_{0}^{\dagger}$, we may have $P \neq P^{\dagger}$, depending on the choice of $V_{1}$. The superpotentials should also be chosen in such a way that, first of all, $Q, P, Q^{\prime}$, and $P^{\prime}$ satisfy the same commutation rules (3.5) as their 0-counterparts:

$$
[Q, P]=\left[Q^{\prime}, P^{\prime}\right]=i, \quad\left[Q, P^{\prime}\right]=\left[Q^{\prime}, P\right]=\left[Q, Q^{\prime}\right]=\left[P, P^{\prime}\right]=0 .
$$

These impose certain conditions on $\vec{V}$ and $\vec{W}$ :

$$
W_{1, x}=V_{2, y}, \quad W_{2, x}=-V_{2, x}, \quad W_{1, y}=-V_{1, y}, \quad W_{2, y}=V_{1, x},
$$

as well as

$$
V_{1, x}+V_{2, y}=W_{1, x}+W_{2, y}=-1 .
$$

The subscripts $x, y$ denote differentiation with respect to that variable. Hence, as it was already clear at the beginning, the two different vector superpotentials must be related to each other. Notice that the standard choice trivially satisfies all these conditions. We now introduce the following operators:

$$
A^{\prime}=\alpha^{\prime}\left(Q^{\prime}+i P^{\prime}\right), \quad B^{\prime}=\gamma^{\prime}\left(Q^{\prime}-i P^{\prime}\right), \quad A=\alpha(Q+i P), \quad B=\gamma(Q-i P),
$$

where $\alpha \gamma=\frac{1}{2}$ and $\alpha^{\prime} \gamma^{\prime}=\frac{1}{2}$. Incidentally, we recall that for the SLL the same linear combinations as in (3.12) are found with $\alpha=\alpha^{\prime}=\gamma=\gamma^{\prime}=\frac{1}{\sqrt{2}}$ and with the operators $Q, P, Q^{\prime}$, and $P^{\prime}$ replaced, respectively, by $Q_{0}, P_{0}, Q_{0}^{\prime}$, and $P_{0}^{\prime}$. Thus, the operators generalizing the Landau Hamiltonians in Ref. 6 are

$$
h^{\prime}=\frac{1}{2}\left(p_{x}+W_{2}\right)^{2}+\frac{1}{2}\left(p_{y}-W_{1}\right)^{2}, \quad h=\frac{1}{2}\left(p_{x}-V_{2}\right)^{2}+\frac{1}{2}\left(p_{y}+V_{1}\right)^{2},
$$

which can be rewritten as

$$
h^{\prime}=B^{\prime} A^{\prime}-\frac{1}{2} 11, \quad h=B A-\frac{1}{2} 11 .
$$

The operators in (3.12) are pseudo-bosonic since they satisfy the following commutation rules:

$$
[A, B]=\left[A^{\prime}, B^{\prime}\right]=\mathbb{1},
$$

while all the other commutators are trivial. It is important to observe that, since $A^{\dagger}=\bar{\alpha}\left(Q^{\dagger}-i P^{\dagger}\right)$, and since $Q$ and $P$ are not necessarily self-adjoint, in general $B \neq A^{\dagger}$. Analogously, in general $B^{\prime} \neq A^{\prime \dagger}$. Similar conclusions can be deduced starting from the pairs $B^{\dagger}, A^{\dagger}$ and $B^{\prime \dagger}, A^{\prime \dagger}$.

At this stage, it is interesting to say few words on the physical meaning of our model. In other words: what is the physical meaning of going from the SLL to these GLLs? The answer is the following: suppose we interpret $\vec{W}$ and $\vec{V}$ in (3.13) as two different (but related) vector potentials describing two possibly different magnetic fields. These potentials are $\vec{A}_{\uparrow}=\left(W_{2},-W_{1}, 0\right)$ for $h^{\prime}$ 
and $\vec{A}_{\downarrow}=\left(-V_{2}, V_{1}, 0\right)$ for $h$ (the reason for this notation will be clear in a moment). Now, computing the associated magnetic fields from these vectors, we get

$$
\vec{B}_{\uparrow}=\vec{\nabla} \wedge \vec{A}_{\uparrow}=-\hat{k}\left(\partial_{x} W_{1}+\partial_{y} W_{2}\right)=\hat{k}, \quad \vec{B}_{\downarrow}=\vec{\nabla} \wedge \vec{A}_{\downarrow}=\hat{k}\left(\partial_{x} V_{1}+\partial_{y} V_{2}\right)=-\hat{k},
$$

because of the equalities in (3.11). Hence, for any possible choice of superpotentials, $h^{\prime}$ and $h$, respectively, describe an electron in an up and in a down uniform magnetic field, as the original Hamiltonians $H_{0}^{\prime}$ and $H_{0}$. Incidentally, this suggests that we should further analyze this model in the light of the modular structure, recently considered in Ref. 14 in the context of SLLs.

The following are some possible choices of $\vec{W}$ and $\vec{V}$ :

Choice 1 (SLL). Let us take $V_{1}(x, y)=W_{1}(x, y)=-\frac{x}{2}, \quad V_{2}(x, y)=W_{2}(x, y)=-\frac{y}{2}$. If we further take $\alpha=\gamma=\alpha^{\prime}=\gamma^{\prime}=\frac{1}{\sqrt{2}}$, we recover exactly the usual situation. ${ }^{6}$ Moreover, we go back to bosonic rather than pseudo-bosonic commutation relations.

Choice 2 (Perturbations of the SLL). First, we consider a symmetric perturbation. For that we take $V_{1}(x, y)=-\frac{x}{2}+v_{1}(y), V_{2}(x, y)=-\frac{y}{2}+v_{2}(x)$, where $v_{1}$ and $v_{2}$ are arbitrary (but sufficiently regular) functions. Hence, we get, apart from inessential additive constants, $W_{1}(x, y)=-\frac{x}{2}-v_{1}(y)$, $W_{2}(x, y)=-\frac{y}{2}-v_{2}(x)$. In order not to trivialize the situation, it is also necessary to take $v_{1}(y)$ and $v_{2}(x)$ complex (at least one of them): this is the way to get pseudo-bosons rather than simple bosons.

A nonsymmetric version of this perturbation can be constructed by just taking $V_{1}(x, y)=$ $-a_{1} x+v_{1}(y), V_{2}(x, y)=-a_{2} y+v_{2}(x)$, with $a_{1}+a_{2}=1$.

Choice 3 (A general solution). We take $V_{1}(x, y)=-x+v_{1}(y)+\int \frac{\partial V_{2}(x, y)}{\partial y} d x$, where $V_{2}(x, y)$ is any function for which this definition makes sense. In particular, for instance, if we take $V_{2}(x, y)=e^{x y}$, then $V_{1}(x, y)=-x+v_{1}(y)+\frac{1}{y^{2}}(x y-1) e^{x y}$ and, consequently, $W_{1}(x, y)=$ $-v_{1}(y)-\frac{1}{y^{2}}(x y-1) e^{x y}$ and $W_{2}(x, y)=-y-e^{x y}$.

If we rather take $V_{2}(x, y)=x^{n} y^{k}, n, k=1,2,3, \ldots$, then $V_{1}(x, y)=-x+v_{1}(y)-$ $\frac{k}{n+1} x^{n+1} y^{k-1}$, and so on.

\section{A. A perturbation of the SLL}

We will now focus our attention on Choice 2 above, with an explicit choice of $v_{1}(y)$ and $v_{2}(x)$, and apply the construction given in Sec. II. Let

$$
W_{1}(x, y)=-\frac{x}{2}-i k_{1} y, \quad W_{2}(x, y)=-\frac{y}{2}-i k_{2} x,
$$

with $k_{1}$ and $k_{2}$ real and not both zero (not to go back to SLLs). In this case, the operators in (3.12) assume the following differential expressions:

$$
\left\{\begin{array}{l}
A^{\prime}=\alpha^{\prime}\left(\partial_{x}-i \partial_{y}+\frac{x}{2}\left(1+2 k_{2}\right)-\frac{i y}{2}\left(1-2 k_{1}\right)\right), \\
B^{\prime}=\gamma^{\prime}\left(-\partial_{x}-i \partial_{y}+\frac{x}{2}\left(1-2 k_{2}\right)+\frac{i y}{2}\left(1+2 k_{1}\right)\right), \\
A=\alpha\left(-i \partial_{x}+\partial_{y}-\frac{i x}{2}\left(1+2 k_{2}\right)+\frac{y}{2}\left(1-2 k_{1}\right)\right), \\
B=\gamma\left(-i \partial_{x}-\partial_{y}+\frac{i x}{2}\left(1-2 k_{2}\right)+\frac{y}{2}\left(1+2 k_{1}\right)\right) .
\end{array}\right.
$$

In order to check Assumptions 1 and 2 of the previous section, we first look for vectors $\varphi_{0,0}(x, y)$ and $\Psi_{0,0}(x, y)$ satisfying $A \varphi_{0,0}(x, y)=0$ and $B^{\dagger} \Psi_{0,0}(x, y)=0$. We get

$$
\left\{\begin{array}{l}
\varphi_{0,0}(x, y)=N_{\varphi} \exp \left\{-\frac{x^{2}}{4}\left(1+2 k_{2}\right)-\frac{y^{2}}{4}\left(1-2 k_{1}\right)\right\} \\
\Psi_{0,0}(x, y)=N_{\Psi} \exp \left\{-\frac{x^{2}}{4}\left(1-2 k_{2}\right)-\frac{y^{2}}{4}\left(1+2 k_{1}\right)\right\},
\end{array}\right.
$$

where $N_{\varphi}$ and $N_{\Psi}$ are normalization constants, which are chosen in such a way that $\left\langle\varphi_{0,0}, \Psi_{0,0}\right\rangle=1$. Of course, in order for this result to make sense, the two functions must belong to the Hilbert space $\mathcal{H}$ we are considering here, i.e., $\mathcal{L}^{2}\left(\mathbb{R}^{2}\right)$. This imposes some constraints on $k_{1}$ and $k_{2}:-\frac{1}{2}<k_{j}<\frac{1}{2}$, $j=1,2$.

It is possible to check that the same functions also satisfy $A^{\prime} \varphi_{0,0}(x, y)=0$ and $B^{\prime \dagger} \Psi_{0,0}(x, y)=0$. It is now evident that Assumptions 1 and 2 are satisfied. Indeed, the action of, 
say, $B_{1}^{n}$ on $\varphi_{0,0}(x, y)$ simply produces some polynomial [see (3.21) below] of the $n$th degree times a Gaussian: this resulting function belongs clearly to $\mathcal{L}^{2}\left(\mathbb{R}^{2}\right)$ for all $n$. This fact allows us to define the following functions:

$$
\varphi_{n, l}(x, y)=\frac{B^{\prime n} B^{l}}{\sqrt{n ! l !}} \varphi_{0,0}(x, y), \quad \text { and } \quad \Psi_{n, l}(x, y)=\frac{\left(A^{\prime \dagger}\right)^{n}\left(A^{\dagger}\right)^{l}}{\sqrt{n ! l !}} \Psi_{0,0}(x, y),
$$

where $n, l=0,1,2,3, \ldots$ As we have seen in the previous section, the sets $\mathcal{F}_{\Psi}=\left\{\Psi_{n, l}(x, y), n\right.$, $l \geq 0\}$ and $\mathcal{F}_{\varphi}=\left\{\varphi_{n, l}(x, y), n, l \geq 0\right\}$ are biorthogonal. In fact, with our previous choice of the normalization constants, we have

$$
\left\langle\Psi_{n, l}, \varphi_{m, k}\right\rangle=\delta_{n, m} \delta_{l, k}, \quad \forall n, m, l, k \geq 0 .
$$

Of course these vectors diagonalize the operators $h=N-\frac{1}{2} \mathbb{1 1}$ and $h^{\prime}=N^{\prime}-\frac{1}{2} \mathbb{1}$, as well as their adjoints $h^{\dagger}=\mathfrak{N}-\frac{1}{2} \mathbb{1 1}$ and $h^{\prime \dagger}=\mathfrak{N}^{\prime}-\frac{1}{2} \mathbb{1}$, where $N=B A, N^{\prime}=B^{\prime} A^{\prime}, \mathfrak{N}=N^{\dagger}$ and $\mathfrak{N}^{\prime}=N^{\prime \dagger}$. We find

$$
h^{\prime} \varphi_{n, l}=\left(n-\frac{1}{2}\right) \varphi_{n, l}, \quad h \varphi_{n, l}=\left(l-\frac{1}{2}\right) \varphi_{n, l}
$$

and

$$
h^{\prime \dagger} \Psi_{n, l}=\left(n-\frac{1}{2}\right) \Psi_{n, l}, \quad h^{\dagger} \Psi_{n, l}=\left(l-\frac{1}{2}\right) \Psi_{n, l} .
$$

The next step consists in proving that the sets $\mathcal{F}_{\varphi}$ and $\mathcal{F}_{\Psi}$ are complete in $\mathcal{H}$. This is a consequence of the fact that (i) the set $\mathcal{F}_{h}:=\left\{h_{n, m}(x, y):=x^{n} y^{m} \varphi_{0,0}(x, y), n, m \geq 0\right\}$ is complete in $\mathcal{L}^{2}\left(\mathbb{R}^{2}\right)$; (ii) each function of $\mathcal{F}_{h}$ can be written as a finite linear combination of some $\varphi_{i, j}(x, y)$. Then it is clear that, if by assumption $f \in \mathcal{H}$ is such that $\left\langle f, \varphi_{i, j}\right\rangle=0$ for all $i$ and $j$, then $\left\langle f, h_{n, m}\right\rangle=0$ for all $n$ and $m$, so that $f=0$. Of course the same argument allows us to prove that $\mathcal{F}_{\Psi}$ is complete in $\mathcal{H}$.

This result implies that also Assumption 3 of Sec. II is satisfied. Now we could introduce the intertwining operators $S_{\varphi}$ and $S_{\Psi}$ and check, among other properties, if they are bounded or not. This is related to the fact that, as we will first show, the sets $\mathcal{F}_{\varphi}$ and $\mathcal{F}_{\Psi}$ are not Riesz bases, except when $k_{1}=k_{2}=0$ [see (3.18)]. To check this claim, we introduce the orthonormal basis of $\mathcal{L}^{2}\left(\mathbb{R}^{2}\right)$ arising from the SLL, ${ }^{6}$

$$
\mathcal{F}_{\varphi}^{(0)}:=\left\{\varphi_{n, l}^{(0)}(x, y):=\frac{B_{0}^{\prime n} B_{0}^{l}}{\sqrt{n ! l !}} \varphi_{0,0}^{(0)}(x, y), \quad n, m \geq 0\right\}
$$

where $\varphi_{0,0}^{(0)}(x, y)=\frac{1}{\sqrt{2 \pi}} e^{-\left(x^{2}+y^{2}\right) / 4}$ is the vacuum of $A_{0}=\frac{1}{\sqrt{2}}\left(Q_{0}+i P_{0}\right)$ and $A_{0}^{\prime}=\frac{1}{\sqrt{2}}\left(Q_{0}^{\prime}+i P_{0}^{\prime}\right)$. Recall that, for SLLs, $B_{0}^{\prime}=A_{0}^{\prime \dagger}$ and $B_{0}=A_{0}^{\dagger}$.

To prove now that $\mathcal{F}_{\varphi}$ is not a Riesz basis, we will show that an operator $T_{\varphi}$ exists mapping $\mathcal{F}_{\varphi}^{(0)}$ into $\mathcal{F}_{\varphi}$, that $T_{\varphi}$ is invertible, but $T_{\varphi}$ and/or $T_{\varphi}^{-1}$ are not bounded. Finding this operator is simple. Indeed, it is easy to first check that

$$
\varphi_{n, 0}^{(0)}(x, y)=\frac{1}{\sqrt{2^{n} n !}}(x+i y)^{n} \varphi_{0,0}^{(0)}(x, y), \quad \varphi_{0, l}^{(0)}(x, y)=\frac{i^{l}}{\sqrt{2^{l} l !}}(x-i y)^{l} \varphi_{0,0}^{(0)}(x, y)
$$

and

$$
\varphi_{n, 0}(x, y)=\frac{\gamma^{\prime n}}{\sqrt{n !}}(x+i y)^{n} \varphi_{0,0}(x, y), \quad \varphi_{0, l}(x, y)=\frac{(i \gamma)^{l}}{\sqrt{l !}}(x-i y)^{l} \varphi_{0,0}(x, y),
$$

for all $n, l \geq 0$. Similar formulae are deduced for $\Psi_{n, 0}(x, y)$ and $\Psi_{0, l}(x, y)$. From a comparison between (3.21) and (3.22), it is clear that $T_{\varphi}$ can exist only if $\gamma=\gamma^{\prime}=\frac{1}{\sqrt{2}}$. Assuming this to be so, we have

$$
\frac{\varphi_{n, 0}(x, y)}{\varphi_{n, 0}^{(0)}(x, y)}=\frac{\varphi_{0, l}(x, y)}{\varphi_{0, l}^{(0)}(x, y)}=\frac{\varphi_{0,0}(x, y)}{\varphi_{0,0}^{(0)}(x, y)}
$$


for all $n, l \geq 0$. This suggest that we define $T_{\varphi}$ as the ratio in the right-hand side of this equality:

$$
T_{\varphi}=\frac{\varphi_{0,0}(x, y)}{\varphi_{0,0}^{(0)}(x, y)}=\sqrt{2 \pi} N_{\varphi} e^{-\frac{x^{2}}{2} k_{2}+\frac{y^{2}}{2} k_{1}} .
$$

Of course, we have still to check that with this definition $\varphi_{n, l}(x, y)=T_{\varphi} \varphi_{n, l}^{(0)}(x, y)$ holds also if both $n$ and $l$ are not zero. This can be proved by observing that, for all $n \geq 0$, the following intertwining relation holds:

$$
B^{\prime n} T_{\varphi}=T_{\varphi}\left(A_{0}^{\prime \dagger}\right)^{n}
$$

Therefore, since

$$
\varphi_{n, l}(x, y)=T_{\varphi} \varphi_{n, l}^{(0)}(x, y) \quad \Leftrightarrow \quad B^{\prime n} \varphi_{0, l}=T_{\varphi}\left(A_{0}^{\prime \dagger}\right)^{n} \varphi_{0, l}^{(0)} \quad \Leftrightarrow \quad B^{\prime n} T_{\varphi} \varphi_{0, l}^{(0)}=T_{\varphi}\left(A_{0}^{\prime \dagger}\right)^{n} \varphi_{0, l}^{(0)},
$$

our claim immediately follows. Formula (3.25) can be proved by induction on $n$. The inverse of $T_{\varphi}$ is $T_{\varphi}^{-1}=\frac{1}{\sqrt{2 \pi} N_{\varphi}} e^{\frac{x^{2}}{2} k_{2}-\frac{y^{2}}{2} k_{1}}$. It is clear that both $T_{\varphi}$ and/or $T_{\varphi}^{-1}$ are unbounded on $\mathcal{L}^{2}\left(\mathbb{R}^{2}\right)$ for all possible choices of $k_{1}$ and $k_{2}$ in $\left(-\frac{1}{2}, \frac{1}{2}\right)$, except when $k_{1}=k_{2}=0$, i.e., in the case of the SLL. Hence, for well known general reasons, ${ }^{15,16} \mathcal{F}_{\varphi}$ cannot be a Riesz basis.

Essentially, the same arguments also show that $\mathcal{F}_{\Psi}$ is not a Riesz basis, either. Indeed, an operator $T_{\Psi}$ mapping $\mathcal{F}_{\varphi}^{(0)}$ into $\mathcal{F}_{\Psi}$ can be found and it is

$$
T_{\Psi}=\frac{\Psi_{0,0}(x, y)}{\varphi_{0,0}^{(0)}(x, y)}=\sqrt{2 \pi} N_{\Psi} e^{\frac{x^{2}}{2} k_{2}-\frac{y^{2}}{2} k_{1}} .
$$

This operator satisfies $\Psi_{n, l}(x, y)=T_{\Psi} \varphi_{n, l}^{(0)}(x, y)$ for all possible choices of $n$ and $l$ greater or equal to zero. Therefore, since $\varphi_{n, l}(x, y)=T_{\varphi} \varphi_{n, l}^{(0)}(x, y)=\left(T_{\varphi} T_{\Psi}^{-1}\right) \Psi_{n, l}(x, y)$, the operators $S_{\varphi}$ and $S_{\Psi}$ in (2.6) can be easily identified and look like

$$
S_{\varphi}=T_{\varphi} T_{\Psi}^{-1}=\frac{N_{\varphi}}{N_{\Psi}} e^{-x^{2} k_{2}+y^{2} k_{1}}, \quad S_{\Psi}=S_{\varphi}^{-1}=T_{\Psi} T_{\varphi}^{-1}=\frac{N_{\Psi}}{N_{\varphi}} e^{x^{2} k_{2}-y^{2} k_{1}}
$$

Notice that for any choice of $k_{1}$ and $k_{2}$ in $\left(-\frac{1}{2}, \frac{1}{2}\right)$, other than when $\left(k_{1}, k_{2}\right)=(0,0)$, at least one of these operators is unbounded.

We will now construct a set of bicoherent states for our GLL. However, rather than using the definitions in (2.12), it is convenient to look for solutions in the $(x, y)$-space of the eigenvalue equations

$$
\left\{\begin{array}{l}
A \tilde{\varphi}\left(x, y ; z, z^{\prime}\right)=z \tilde{\varphi}\left(x, y ; z, z^{\prime}\right) \\
A^{\prime} \tilde{\varphi}\left(x, y ; z, z^{\prime}\right)=z^{\prime} \tilde{\varphi}\left(x, y ; z, z^{\prime}\right) \\
B^{\dagger} \tilde{\Psi}\left(x, y ; z, z^{\prime}\right)=z \tilde{\Psi}\left(x, y ; z, z^{\prime}\right) \\
B^{\prime \dagger} \tilde{\Psi}\left(x, y ; z, z^{\prime}\right)=z^{\prime} \tilde{\Psi}\left(x, y ; z, z^{\prime}\right)
\end{array}\right.
$$

where, as suggested by our previous results, we take $\alpha=\alpha^{\prime}=\gamma=\gamma^{\prime}=\frac{1}{\sqrt{2}}$ in (3.17). The square integrable solutions of the differential equations in (3.28) are

$$
\left\{\begin{array}{l}
\tilde{\varphi}\left(x, y ; z, z^{\prime}\right)=N_{A}\left(z, z^{\prime}\right) e^{-\left[\left(1+2 k_{2}\right) x^{2}-\left(1-2 k_{1}\right) y^{2}\right] / 4} e^{\frac{1}{\sqrt{2}}\left[\left(z^{\prime}+i z\right) x+\left(z+i z^{\prime}\right) y\right]} \\
\tilde{\Psi}\left(x, y ; z, z^{\prime}\right)=N_{B}\left(z, z^{\prime}\right) e^{-\left[\left(1-2 k_{2}\right) x^{2}-\left(1+2 k_{1}\right) y^{2}\right] / 4} e^{\frac{1}{\sqrt{2}}\left[\left(z^{\prime}+i z\right) x+\left(z+i z^{\prime}\right) y\right]},
\end{array}\right.
$$

where $z$ and $z^{\prime}$ are complex parameters.

The normalization is fixed by requiring that

$$
\left\langle\tilde{\varphi}\left(x, y ; z, z^{\prime}\right), \tilde{\Psi}\left(x, y ; z, z^{\prime}\right)\right\rangle_{\mathcal{L}^{2}\left(\mathbb{R}^{2}\right)}=\left\langle\varphi\left(z, z^{\prime}\right), \Psi\left(z, z^{\prime}\right)\right\rangle_{\mathcal{H}},
$$

where in the right-hand side the coherent states introduced in (2.12), and living in the Hilbert space $\mathcal{H}$, appear. Notice that $\left\langle\varphi\left(z, z^{\prime}\right), \Psi\left(z, z^{\prime}\right)\right\rangle_{\mathcal{H}}=1$ for all $z$ and $z^{\prime}$ in $\mathcal{D}$. Then, we find, with a suitable choice of phases,

$$
N_{A}\left(z, z^{\prime}\right) N_{B}\left(z, z^{\prime}\right)=\frac{1}{2 \pi} e^{-\left|z-i \bar{z}^{\prime}\right|^{2}}
$$


Notice that these states reduce to the standard 2D Gaussian $\frac{1}{\sqrt{2 \pi}} e^{-\left(x^{2}+y^{2}\right) / 4}$ when $z=z^{\prime}=k_{1}=$ $k_{2}=0$, i.e., for the SLL and for eigenvalues of the lowering operators, both equal to zero. It is now a straightforward computation to check the resolution of the identity for these states

$$
\frac{1}{\pi^{2}} \int_{\mathbb{C}^{2}} d z d z^{\prime}\left|\tilde{\varphi}\left(x, y ; z, z^{\prime}\right)><\tilde{\Psi}\left(x, y ; z, z^{\prime}\right)\right|=\mathbb{1}
$$

where 11 is the identity in $\mathcal{L}^{2}\left(\mathbb{R}^{2}\right)$.

This result is by no means in disagreement with the theorem stated in Sec. II. The first reason is that it is not clear that the functions $\tilde{\varphi}\left(x, y ; z, z^{\prime}\right)$ and $\tilde{\Psi}\left(x, y ; z, z^{\prime}\right)$ coincide with $\varphi\left(z, z^{\prime}\right)$ and $\Psi\left(z, z^{\prime}\right)$, for which the theorem was stated. The second, and more important, reason is that the theorem gives only a sufficient condition. Hence, if we would be able to prove that $\tilde{\varphi}\left(x, y ; z, z^{\prime}\right)=\varphi\left(z, z^{\prime}\right)$ and $\tilde{\Psi}\left(x, y ; z, z^{\prime}\right)=\Psi\left(z, z^{\prime}\right)$, this computation will provide a nice counterexample showing that the conditions of the theorem are, in fact, only sufficient and not necessary. This is work in progress.

\section{DAMPED HARMONIC OSCILLATOR}

An interesting example of $2 \mathrm{D}$ pseudo-bosons is provided by the damped harmonic oscillator (DHO). In Ref. 7, the authors have discussed a possible approach to the quantization of the DHO. This is a nonconservative system, so that a Hamiltonian approach requires a certain amount of care. The approach, which was proposed already in $1977,{ }^{17}$ is to consider the DHO as a part of a larger system, involving also a second oscillator, which is forced and which takes the energy lost by the DHO, so that this larger system is conservative. The original equation of motion, $m \ddot{x}+\gamma \dot{x}+k x=0$, is therefore complemented by a second virtual equation, $m \ddot{y}-\gamma \dot{y}+k y=0$, and the classical lagrangian for the system looks like $L=m \dot{x} \dot{y}+\frac{\gamma}{2}(x \dot{y}-\dot{x} y)-k x y$, which corresponds to a classical Hamiltonian $H=p_{x} \dot{x}+p_{y} \dot{y}-L=\frac{1}{m}\left(p_{x}+\gamma \frac{y}{2}\right)\left(p_{y}-\gamma \frac{x}{2}\right)+k x y$, where $p_{x}=\frac{\partial L}{\partial \dot{x}}$ and $p_{y}=\frac{\partial L}{\partial \dot{y}}$ are the conjugate momenta. The introduction of pseudo-bosons is based on two successive changes of variables and on a canonical quantization. First of all, we introduce the new variables $x_{1}$ and $x_{2}$ via $x=\frac{1}{\sqrt{2}}\left(x_{1}+x_{2}\right), y=\frac{1}{\sqrt{2}}\left(x_{1}-x_{2}\right)$. Then $L=\frac{1}{2} m\left(\dot{x}_{1}^{2}-\dot{x}_{2}^{2}\right)+\frac{\gamma}{2}\left(x_{2} \dot{x}_{1}-\right.$ $\left.x_{1} \dot{x}_{2}\right)-\frac{k}{2}\left(x_{1}^{2}-x_{2}^{2}\right)$ and $H=\frac{1}{2 m}\left(p_{1}-\gamma \frac{x_{2}}{2}\right)^{2}+\frac{1}{2 m}\left(p_{2}+\gamma \frac{x_{1}}{2}\right)^{2}+\frac{k}{2}\left(x_{1}^{2}-x_{2}^{2}\right)$. The second change of variable is the following:

$$
\left\{\begin{array}{l}
p_{+}=\sqrt{\frac{\omega_{+}}{2 m \Omega}} p_{1}+i \sqrt{\frac{m \Omega \omega_{+}}{2}} x_{2}, \\
p_{-}=\sqrt{\frac{\omega_{-}}{2 m \Omega}} p_{1}-i \sqrt{\frac{m \Omega \omega_{-}}{2}} x_{2}, \\
x_{+}=\sqrt{\frac{m \Omega}{2 \omega_{+}}} x_{1}+i \sqrt{\frac{1}{2 m \Omega \omega_{+}}} p_{2}, \\
x_{-}=\sqrt{\frac{m \Omega}{2 \omega_{-}}} x_{1}-i \sqrt{\frac{1}{2 m \Omega \omega_{-}}} p_{2},
\end{array}\right.
$$

where we have introduced $\Omega=\sqrt{\frac{1}{m}\left(k-\frac{\gamma^{2}}{4 m}\right)}$ and the two following complex quantities $\omega_{ \pm}=$ $\Omega \pm i \frac{\gamma}{2 m}$. In the rest of the section, we will assume that $k \geq \frac{\gamma^{2}}{4 m}$, so that $\Omega$ is real. Up to now, we are still at a classical level, so that $\bar{\omega}_{+}=\omega_{-}, \bar{p}_{+}=p_{-}, \bar{x}_{+}=x_{-}$, and consequently, see below, $\bar{H}_{+}=H_{-}$ and $\bar{H}=H$. Hence, $H$ is a real Hamiltonian. Indeed, with these definitions, the Hamiltonian looks like the Hamiltonian of a 2D harmonic oscillator

$$
H=\frac{1}{2}\left(p_{+}^{2}+\omega_{+}^{2} x_{+}^{2}\right)+\frac{1}{2}\left(p_{-}^{2}+\omega_{-}^{2} x_{-}^{2}\right)=: H_{+}+H_{-}
$$

at least formally.

At this stage, we quantize canonically the system $^{7}$ : we require that the following commutators are satisfied:

$$
\left[x_{+}, p_{+}\right]=\left[x_{-}, p_{-}\right]=i 1,
$$

all the other commutators being trivial. We also have to require that $p_{+}^{\dagger}=p_{-}$and that $x_{+}^{\dagger}=x_{-}$, which are the quantum version of the compatibility conditions above. The pseudo-bosons now 
appear:

$$
\left\{\begin{array}{l}
a_{+}=\sqrt{\frac{\omega_{+}}{2}}\left(x_{+}+i \frac{p_{+}}{\omega_{+}}\right), \\
a_{-}=\sqrt{\frac{\omega_{-}}{2}}\left(x_{-}+i \frac{p_{-}}{\omega_{-}}\right), \\
b_{+}=\sqrt{\frac{\omega_{+}}{2}}\left(x_{+}-i \frac{p_{+}}{\omega_{+}}\right), \\
b_{-}=\sqrt{\frac{\omega_{-}}{2}}\left(x_{-}-i \frac{p_{-}}{\omega_{-}}\right),
\end{array}\right.
$$

and indeed we have $\left[a_{+}, b_{+}\right]=\left[a_{-}, b_{-}\right]=11$, all the other commutators being zero. Notice also that $b_{+}=a_{-}^{\dagger}$ and $b_{-}=a_{+}^{\dagger}$. Moreover, $H$ can be written in term of the operators $N_{ \pm}=b_{ \pm} a_{ \pm}$as $H=\omega_{+} N_{+}+\omega_{-} N_{-}+\frac{\omega_{+}+\omega_{-}}{2} 11$. So, the Hamiltonian of the quantum DHO is simply written in terms of pseudo-bosonic operators.

\section{A. About Assumptions 1-3}

This system provides a nontrivial example of pseudo-bosonic operators, which do not satisfy any of the Assumptions 1-3 of Sec. II. To show this, we first observe that a possible representation of the operators in (4.2) is the following:

$$
\left\{\begin{array}{l}
x_{+}=\frac{1}{\Gamma \bar{\delta}-\delta \bar{\Gamma}}\left(\bar{\Gamma} p_{y}+\bar{\delta} x\right) \\
x_{-}=\frac{-1}{\Gamma \bar{\delta}-\delta \bar{\Gamma}}\left(\Gamma p_{y}+\delta x\right) \\
p_{+}=\Gamma p_{x}+\delta y \\
p_{-}=\bar{\Gamma} p_{x}+\bar{\delta} y
\end{array}\right.
$$

for all choices of $\Gamma$ and $\delta$ such that $\Gamma \bar{\delta} \neq \delta \bar{\Gamma}$. Here, $x, y, p_{x}$ and $p_{y}$ are pairwise conjugate self-adjoint operators: $\left[x, p_{x}\right]=\left[y, p_{y}\right]=i$ ll. Notice that these operators also satisfy the compatibility conditions $p_{+}^{\dagger}=p_{-}$and $x_{+}^{\dagger}=x_{-}$. Hence it is natural to represent $x$ and $y$ as the standard multiplication operators and $p_{x}$ and $p_{y}$ as $-i \frac{\partial}{\partial x}=-i \partial_{x}$ and $-i \frac{\partial}{\partial y}=-i \partial_{y}$. Then, we get

$$
\left\{\begin{array}{l}
a_{+}=\sqrt{\frac{\omega_{+}}{2}}\left\{\left(\beta x+i \frac{\delta}{\omega_{+}} y\right)+\left(\frac{\Gamma}{\omega_{+}} \partial_{x}-i \alpha \partial_{y}\right)\right\}, \\
a_{-}=\sqrt{\frac{\omega_{-}}{2}}\left\{\left(\bar{\beta} x+i \frac{\bar{\delta}}{\omega_{-}} y\right)+\left(\frac{\bar{\Gamma}}{\omega_{-}} \partial_{x}-i \bar{\alpha} \partial_{y}\right)\right\}, \\
b_{+}=\sqrt{\frac{\omega_{+}}{2}}\left\{\left(\beta x-i \frac{\delta}{\omega_{+}} y\right)-\left(\frac{\Gamma}{\omega_{+}} \partial_{x}+i \alpha \partial_{y}\right)\right\}, \\
b_{-}=\sqrt{\frac{\omega_{-}}{2}}\left\{\left(\bar{\beta} x-i \frac{\bar{\delta}}{\omega_{-}} y\right)-\left(\frac{\bar{\Gamma}}{\omega_{-}} \partial_{x}+i \bar{\alpha} \partial_{y}\right)\right\}
\end{array}\right.
$$

where, to simplify the notation, we have introduced $\alpha=\frac{\bar{\Gamma}}{\Gamma \bar{\delta}-\delta \bar{\Gamma}}$ and $\beta=\frac{\bar{\delta}}{\Gamma \bar{\delta}-\delta \bar{\Gamma}}$.

Remark. a different representation of $x_{ \pm}$and $p_{ \pm}$could be deduced using the results of Sec. III. However, while the pseudo-bosonic commutation rules would be easily recovered, the compatibility conditions $x_{+}^{\dagger}=x_{-}$and $p_{+}^{\dagger}=p_{-}$would be lost. Hence, this choice is not compatible with our requirements.

Assumption 1 of Sec. II requires the existence of a square-integrable function $\varphi_{0,0}(x, y)$ such that, first of all, $a_{+} \varphi_{0,0}(x, y)=a_{-} \varphi_{0,0}(x, y)=0$. Analogously, Assumption 2 requires the existence of a (possibly different) square-integrable function $\Psi_{0,0}(x, y)$ such that, first of all, $b_{+}^{\dagger} \Psi_{0,0}(x, y)=$ $b_{-}^{\dagger} \Psi_{0,0}(x, y)=0$. However, since $b_{+}=a_{-}^{\dagger}$ and $b_{-}=a_{+}^{\dagger}$, these two functions, if they exist, satisfy the same differential equations. Hence, apart from a normalization constant, we can chose them to be coincident. It is possible to check that a solution of $a_{+} \varphi_{0,0}(x, y)=a_{-} \varphi_{0,0}(x, y)=0$ is the following:

$$
\varphi_{0,0}(x, y)=N_{0} \exp \left\{-\frac{\beta \omega_{+}}{2 \Gamma} x^{2}+\frac{\delta}{2 \alpha \omega_{+}} y^{2}\right\} .
$$


Notice that, in order for this function to be a solution of both $a_{+} \varphi_{0,0}(x, y)=0$ and $a_{-} \varphi_{0,0}(x, y)=0$, it is necessary and sufficient to have the following identity satisfied: $\frac{\omega_{+}}{\omega_{-}}=-\frac{\delta}{\bar{\delta}} \frac{\Gamma}{\bar{\Gamma}}$. This is not a big requirement, clearly. What is crucial, on the other hand, is that the function $\varphi_{0,0}(x, y)$, and $\Psi_{0,0}(x, y)$ should consequently be square integrable. This is possible only if $\Re\left(\frac{\beta \omega_{+}}{2 \Gamma}\right)>0$ and if, at the same time, $\Re\left(\frac{\delta}{\alpha \omega_{+}}\right)<0$. Now, it is not hard to check that these two conditions are incompatible: if one is verified, the other is not. Therefore, the conclusion is that, following the procedure we have considered so far, Assumptions 1 and 2 are violated and, of course, Assumption 3 cannot even be considered since it is meaningless. Of course, this does not mean that for the quantum DHO, the construction proposed in Sec. II cannot be considered. It only means that with the choices we have considered here, this is not possible. It could be possible, however, to look for some different representation of the operators, satisfying the compatibility condition, and see if it is possible to satisfy Assumptions 1-3. This is work in progress.

\section{CONCLUSIONS}

In this paper, we have constructed a physically motivated 2D family of pseudo-bosons arising from a generalized version of the Landau levels. This generalization has been shown to be essentially a gauge transformation. Coherent states have been constructed and the resolution of the identity has been proved.

We have also considered a quantum damped harmonic oscillator: this provides a nice example of a pseudo-bosonic system for which all the assumptions of Sec. II are violated. In conclusion, many examples exist, see Sec. III and Refs. 1,2,5, and 18 among the others, in which Assumptions 1-3, and sometimes Assumption 4, are satisfied. But other examples exist as well for which, even if pseudo-bosonic commutation rules are recovered, none of the Assumptions hold true. This suggests to take care explicitly of these Assumptions when dealing with pseudo-bosons.

\section{ACKNOWLEDGMENTS}

The authors would like to acknowledge financial support from the Università di Palermo through Bando CORI, cap. B.U. 9.3.0002.0001.0001. One of us (STA) would like to acknowledge a grant from the Natural Sciences and Engineering Research Council (NSERC) of Canada.

${ }^{1}$ F. Bagarello, J. Math. Phys. 50, 023531 (2010).

${ }^{2}$ F. Bagarello, J. Math. Phys. 51, 023531 (2010).

${ }^{3}$ F. Bagarello, J. Phys. A 43, 175203 (2010).

${ }^{4}$ F. Bagarello, Examples of pseudo-bosons in quantum mechanics, Phys. Lett. A, in press.

${ }^{5}$ D.A. Trifonov, Pseudo-boson coherent and Fock states, quant-ph/0902.3744.

${ }^{6}$ S. T. Ali and F. Bagarello, J. Math Phys. 49, (2008).

${ }^{7}$ R. Banerjee and P. Mukherjee, J. Phys. A 35, 5591 (2002).

${ }^{8}$ S. Kuru, A. Tegmen, and A. Vercin, J. Math. Phys. 42, 3344 (2001); S. Kuru, B. Demircioglu and M. Onder, and A. Vercin, J. Math. Phys. 43, 2133 (2002); K. A. Samani and M. Zarei, Ann. Phys. 316, 466 (2005).

${ }^{9}$ F. Bagarello, Phys. Lett. A. 372, 6226 (2008); F. Bagarello, J. Phys. A. 42, 075302 (2009); F. Bagarello, J. Math. Phys. 50, 043509 (2009).

${ }^{10}$ A. Mostafazadeh, e-print arXiv:quant-ph/0810.5643; C. Bender, Rep. Progr. Phys., 70, 947 (2007).

${ }^{11}$ S. T. Ali, J.-P. Antoine, and J.-P. Gazeau, Coherent States, Wavelets and Their Generalizations (Springer-Verlag, New York, 2000).

12 J.-P. Gazeau, Coherent States in Quantum Physics (Wiley-VCH, Berlin, 2009).

${ }^{13}$ J. P. Antoine and F. Bagarello, "Localization Properties and Wavelet-Like Orthonormal Bases for the Lowest Landau Level", in Advances in Gabor Analysis, edited by H. G. Feichtinger and T. Strohmer (Birkhäuser, Boston, 2003).

${ }^{14}$ S. T. Ali, F. Bagarello, and G. Honnouvo, J. Phys. A 43, 105202 (2010).

${ }^{15}$ R. Young, An Introduction to Nonharmonic Fourier Series (Academic, New York, 1980).

${ }^{16}$ O. Christensen, An Introduction to Frames and Riesz Bases (Birkhäuser, Boston, 2003).

${ }^{17}$ H. Feshbach and Y. Tikochinsky, N.Y. Acad. Sci., 38, 44 (1977).

${ }^{18}$ F. Bagarello, Phys. Lett. A 374, 3823 (2010).

${ }^{19}$ Recall that a set of vectors $\phi_{1}, \phi_{2}, \phi_{3}, \ldots$, is a Riesz basis of a Hilbert space $\mathcal{H}$, if there exists a bounded operator $V$, with bounded inverse, on $\mathcal{H}$, and an orthonormal basis of $\mathcal{H}, \varphi_{1}, \varphi_{2}, \varphi_{3}, \ldots$, such that $\phi_{j}=V \varphi_{j}$, for all $j=1,2,3, \ldots$ 